\title{
Factorization of Unitary Matrices
}

\author{
P. Diţă \\ Institute of Physics and Nuclear Engineering, \\ P.O. Box MG6, Bucharest, Romania
}

\begin{abstract}
Factorization of an $n \times n$ unitary matrix as a product of $n$ diagonal matrices containing only phases interlaced with $n-1$ orthogonal matrices each one generated by a real vector as well as an explicit form for the Weyl factorization are found.
\end{abstract}

${ }^{0}$ Electronic mail: dita@hera.theory.nipne.ro 


\section{Introduction}

Matrix factorization is a live subject of linear algebra. It seems that no general theory is yet available although many results appear almost every day. However our goal will not be so ambitious to present a general theory of matrix factorizations but to tackle the problem of factorization of unitary matrices. Unitary matrices are a first hand tool in solving many problems in mathematical and theoretical physics and the diversity of the problems necessitates to keep improving it. In fact the matrix factorization is closely related to the parametrization of unitary matrices and the classical result by Murnagham on parametrization of the $n$-dimensional unitary group $U(n)$ is the following: an arbitrary $n \times n$ unitary matrix is the product of a diagonal matrix containing $n$ phases and of $n(n-1) / 2$ matrices whose main building block has the form

$$
U=\left(\begin{array}{cc}
\cos \theta & -\sin \theta e^{-i \varphi} \\
\sin \theta e^{i \varphi} & \cos \theta
\end{array}\right)
$$

The parameters entering the parametrization are $n(n-1) / 2$ angles $\theta_{i}$ and $n(n+$ 1) $/ 2$ phases $\varphi_{i}$. For more details see [1].

A selection of a specific set of angles and/or phases has no theoretical significance because all the choices are mathematically equivalent; however a clever choice may shed some light on important qualitative issues. This is the point of view of Harari and Leurer [2] who recommended a standard choice of the Cabbibo angles and Kobayashi-Maskawa phases for an arbitrary number of quark generations and accordingly they propose a new parametrization (factorization) which, up to a columns permutation, is nothing else than Murnagham parametrization with the change $\theta \rightarrow \pi / 2-\theta$ and $\varphi \rightarrow \varphi+\pi$.

However there are some problems that require a more elaborate factorization. To our knowledge one of the first such problems is that raised by Reck et al. [3] who describe an experimental realization of any discrete unitary operator. Such devices will find practical applications in quantum cryptography and in quantum teleportation. Starting from Murnagham parametrization they show that any $n \times n$ unitary matrix $A_{n}$ can be written as a product

$$
A_{n}=B_{n} C_{n-1}
$$

where $B_{n} \in U(n)$ is at its turn a product of $n-1$ unitary matrices containing each one a block of the form (1) and $C_{n-1}$ is a $U(n-1)$ matrix. Consequently the experimental realization of a $n \times n$ unitary operator is reduced to the realization of two unitary operators out of which one has a lesser dimension. The experimental realization of a $U(3)$ matrix, sketched in Fig. 2 of their paper, suggests that it would be preferable that phases entering the parametrization should be factored out, the device becoming simpler and the phase shifters, their terminology for phases, being placed at the input and output ports respectively. 
A mixing of the Murnagham factorization and that of Reck et al. is that proposed by Rowe et al. [A] in their study on the representations of Weyl group and of Wigner functions for $S U(3)$. The last parametrization is also used by Nemoto in his attempt to develop generalized coherent states for $S U(n)$ systems [5].

Another kind of factorization is that suggested by Chaturvedi and Mukunda in their paper [6] aiming at obtaining a more "suitable" parametrization of the Kobayashi-Maskawa matrix. Although the proposed forms for $n=3,4$ are awfully complicated by comparison with other parametrizations existing in literature, and for this reason it cannot be extended easily to cases $n \geq 5$, the paper contains a novel idea namely that that an $S U(n)$ matrix can be parametrized by a sequence of $n-1$ complex vectors of dimensions $2,3, \ldots, n$. Fortunately there is an alternative construction as it may be inferred from the construction of an $S U(3)$ matrix as a product of two matrices each of them generated by a threeand respectively two-dimensional complex vector [7] in a much more simple form than that presented in ref. [6].

One aim of this paper is to elaborate this alternative construction in order to obtain a parametrization of $n \times n$ unitary matrices as a product of $n$ diagonal matrices containing the phases and $n-1$ orthogonal matrices, each of them generated by a $n$-dimensional real vector. As a byproduct we obtain the Weyl form [8] of a unitary matrix $W=w^{*} d w$ where $w$ is a unitary matrix, $w^{*}$ its adjoint and $d$ a diagonal matrix containing phases. The Weyl factorization was the key ingredient in finding the "radial" part of the Laplace-Beltrami operator on $U(n)$ and $S U(n)$ [9, 10 and this explicit form could help in finding completely the Laplace-Beltrami operator on unitary groups.

The paper is organized as follows: In Sect. 2 we derive a factorization of $n \times n$ unitary matrices as a product of $n$ diagonal matrices interlaced with $n-1$ orthogonal matrices generated by real vectors of dimension $2,3 \ldots, n-1$. The explicit form of orthogonal matrices is found in Sect.3 and the paper ends with Concluding remarks.

\section{Factorization of unitary matrices}

The unitary group $U(n)$ is the group of automorphisms of the Hilbert space $\left(\mathbf{C}^{n},(\cdot, \cdot)\right)$ where $(\cdot, \cdot)$ is the Hermitian scalar product $(x, y)=\sum_{i=1}^{i=n} \overline{x_{i}} y_{i}$. If $A_{n} \in U(n)$ by $A_{n}^{*}$ we will denote the adjoint matrix and then $A_{n}^{*} A_{n}=I_{n}$, where $I_{n}$ is the $n \times n$ unit matrix. It follows that $\operatorname{det} A_{n}=e^{i \varphi}$, where $\varphi$ is a phase, and $\operatorname{dim}_{R} U(n)=n^{2}$.

First of all we want to introduce some notations that will be useful in the following. The product of two unitary matrices being again a unitary matrix it follows that the multiplication of a row or a column by an arbitrary phase does not affect the unitarity property. Indeed the multiplication of the $j^{\text {th }}$ row 
by $e^{i \varphi_{j}}$ is equivalent to the left multiplication by a diagonal matrix whose all diagonal entries but the $j^{\text {th }}$ one are equal to unity and $a_{j j}=e^{i \varphi_{j}}$. The first building blocks appearing in factorization of unitary matrices are diagonal matrices written in the form $d_{n}=\left(e^{i \varphi_{1}}, \ldots, e^{i \varphi_{n}}\right)$ with $\varphi_{j} \in[0,2 \pi), j=1, \ldots, n$ arbitrary phases, and all off-diagonal entries zero. We introduce also the notation $d_{k}^{n-k}=\left(1_{n-k}, e^{i \psi_{1}}, \ldots, e^{i \psi_{k}}\right), k<n$, where $1_{n-k}$ means that the first $(n-k)$ diagonal entries are equal to unity, i.e. it can be obtained from $d_{n}$ by making the first $n-k$ phases zero. Multiplying at left by $d_{n}$ an arbitrary unitary matrix the first row will be multiplied by $e^{i \varphi_{1}}$, the second by $e^{i \varphi_{2}}$, etc. and the last one by $e^{i \varphi_{n}}$. Multiplying at right with $d_{k}^{n-k}$ the first $n-k$ columns remain unmodified and the other ones are multiplied by $e^{i \psi_{1}}, \ldots, e^{i \psi_{k}}$ respectively. A consequence of this property is the following: the phases of the elements of an arbitrary row and/or column can be taken zero or $\pi$ and a convenient choice is to take the elements of first column non-negative numbers less than unity and those of the first row real numbers. This follows from the equivalence between the permutation of the $i^{\text {th }}$ and $j^{\text {th }}$ rows (columns) with the left (right) multiplication by the unitary matrix $P_{i j}$ whose all diagonal entries but $a_{i i}$ and $a_{j j}$ are equal to unity, $a_{i i}=a_{j j}=0, a_{i j}=a_{j i}=1, i \neq j$ and all the other entries vanish. In conclusion an arbitrary $A_{n} \in U(n)$ can be written as a product of two matrices, the first one diagonal, in the form

$$
A_{n}=d_{n} \tilde{A}_{n}
$$

where $\tilde{A}_{n}$ is a matrix with the first column entries non-negative numbers.

Other building blocks that will appear in factorization of $\tilde{A}_{n}$ are the rotations which operate in the $i, i+1$ plane of the form

$$
J_{i, i+1}=\left(\begin{array}{cccc}
I_{i-1} & 0 & 0 \\
0 & \cos \theta_{i} & -\sin \theta_{i} & 0 \\
0 & \sin \theta_{i} & \cos \theta_{i} & \\
0 & 0 & I_{n-i-1}
\end{array}\right), \quad i=1, \ldots, n-1
$$

The above formula contains the block (1) with phase zero unlike other parametrizations [回]-[5], in our parametrization the phases will appear only in diagonal matrices.

Let $v$ be the vector $v=(1,0, \ldots, 0)^{t} \in S_{2 n-1} \in \mathbf{C}^{n}$ where $t$ denotes transpose and $S_{2 n-1}$ is the unit sphere of the Hilbert space $\mathbf{C}^{n}$ whose real dimension is $2 n-1$. By applying $A_{n} \in U(n)$ to the vector $v$ we find

$$
A_{n} v=a=\left(\begin{array}{c}
a_{11} \\
\cdot \\
\cdot \\
\cdot \\
a_{n 1}
\end{array}\right)
$$

where $a \in S_{2 n-1}$ because $A_{n}$ is unitary. The vector $a$ is completely determined by the first column of the matrix $A_{n}$. Conversely, given an arbitrary vector of 
the unit sphere $w \in S_{2 n-1}$ this point determines a unique first row of a unitary matrix which maps $w$ to the vector $v$. Therefore $U(n)$ acts transitively on $S_{2 n-1}$. The subgroup of $U(n)$ which leaves $v$ invariant is $U(n-1)$ on the last $n-1$ dimensions such that

$$
S_{2 n-1}=\text { coset space } U(n) / U(n-1)
$$

A direct consequence of the last relation is that we expect that any element of $U(n)$ should be uniquely specified by a pair of a vector $b \in S_{2 n-1}$ and of an arbitrary element of $U(n-1)$. Thus we are looking for a factorization of an arbitrary element $A_{n} \in U(n)$ in the form

$$
A_{n}=B_{n} \cdot\left(\begin{array}{cc}
1 & 0 \\
0 & A_{n-1}
\end{array}\right)
$$

where $B_{n} \in U(n)$ is a unitary matrix whose first column is uniquely defined by a vector $b \in S_{2 n-1}$, but otherwise still arbitrary and $A_{n-1}$ is an arbitrary element of $U(n-1)$. For the $S U(3)$ group such a factorization was obtained recently [6, 7]. Iterating the previous equation we arrive at the conclusion that an element of $U(n)$ can be written as a product of $n$ unitary matrices

$$
A_{n}=B_{n} \cdot B_{n-1}^{1} \ldots B_{1}^{n-1}
$$

where

$$
B_{n-k}^{k}=\left(\begin{array}{cc}
I_{k} & 0 \\
0 & B_{n-k}
\end{array}\right)
$$

$B_{k}, k=1, \ldots, n-1$, are $k \times k$ unitary matrices whose first column is generated by vectors $b_{k} \in S_{2 k-1}$; for example $B_{1}^{n-1}$ is the diagonal matrix $\left(1, \ldots, 1, e^{i \varphi_{n(n+1)}}\right)$.

The still arbitrary columns of $B_{k}$ will be chosen in such a way that we should obtain a simple form for the matrices $B_{k}^{n-k}$, and we require that $B_{k}$ should be completely specified by the parameters entering the vector $b_{k}$ and nothing else. In the following we show that such a parametrization does exist and then $A_{n} \in U_{n}$ in Eq.(5) will be written as a product of $n \times n$ unitary matrices each one parametrized by $2 k-1, k=1, \ldots, n$, real parameters such that the number of independent parameters entering $A_{n}$ will be $1+3+\cdots+2 n-1=n^{2}$ as it should be.

In other words our problem is to complete an $n \times n$ matrix whose its first column is given by a vector $b_{n} \in S_{2 n-1}$ to a unitary matrix and we have to do it without introducing supplementary parameters. For $n=3$ this was found by us in [11] in an other context and here we give the construction for arbitrary $n$.

If we take into account the property (2) the problem simplifies a little since then

$$
B_{n}=d_{n} \tilde{B}_{n}
$$


where the first column of $\tilde{B}_{n}$ has non-negative entries. Denoting this column by $b_{1}$ we will use the parametrization

$$
b_{1}=\left(\cos \theta_{1}, \cos \theta_{2} \sin \theta_{1}, \ldots, \sin \theta_{1} \ldots \sin \theta_{n-1}\right)^{t}
$$

where $\theta_{i} \in[0, \pi / 2], i=1, \ldots, n-1$; we call $\theta_{i}$ angles. Thus $B_{n}$ will be parametrized by $n$ phases and $n-1$ angles. According to the above factorization $\tilde{B}_{n}$ is nothing else than the orthogonal matrix generated by the vector $b_{1}$. Thus with no loss of generality $B_{n}=d_{n} \mathcal{O}_{n}$ with $\mathcal{O}_{n} \in O(n)$. In this way the factorization of $A_{n}$ will be

$$
A_{n}=d_{n} \mathcal{O}_{n} d_{n-1}^{1} \mathcal{O}_{n-1}^{1} \ldots d_{2}^{n-2} \mathcal{O}_{2}^{n-1} d_{1}^{n-1} I_{n}
$$

where $\mathcal{O}_{n-k}^{k}$ has the same structure as $B_{n-k}^{k}$, i.e

$$
\mathcal{O}_{n-k}^{k}=\left(\begin{array}{cc}
I_{k} & 0 \\
0 & \mathcal{O}_{n-k}
\end{array}\right)
$$

Consequently the factorization of unitary matrices reduces to the parametrization of orthogonal matrices generated by an arbitrary vector of the real $n$-dimensional sphere and in the next section we show how to do it.

\section{Parametrization of orthogonal matrices}

An operator $T$ applying the Hilbert space $\mathcal{H}$ in the Hilbert space $\mathcal{H}^{\prime}$ is a contraction if for any $v \in \mathcal{H}, \quad\|T v\|_{\mathcal{H}^{\prime}} \leq\|v\|_{\mathcal{H}}$, i.e. $\|T\| \leq 1$, [12]. For any contraction we have $T^{*} T \leq I_{\mathcal{H}^{\prime}}$ and $T T^{*} \leq I_{\mathcal{H}}$ and the defect operators

$$
D_{T}=\left(I_{\mathcal{H}}-T^{*} T\right)^{1 / 2}, \quad D_{T^{*}}=\left(I_{\mathcal{H}^{\prime}}-T T^{*}\right)^{1 / 2}
$$

are Hermitian operators in $\mathcal{H}$ and $\mathcal{H}^{\prime}$ respectively. They have the property

$$
T D_{T}=D_{T^{*}} T, \quad T^{*} D_{T^{*}}=D_{T} T^{*}
$$

In the following we are interested in a contraction of a special form, namely that generated by a $n$-dimensional real vector $b \in \mathbf{R}^{\mathbf{n}}$, i.e. $T=\left(b_{1}, \ldots, b_{n}\right)^{t}$, where $b_{i}$ are the coordinates of $b$; its norm is $\|T\|=(b, b)$ and $T$ will be a contraction iff $(b, b) \leq 1$, i.e. if $b$ is a point inside the unit ball of $\mathbf{R}^{n}$. If $(b, b)=1$, that is the case we are interested in, $T$ is an isometry, i.e.

$$
T^{*} T=1, \quad \text { and } \quad D_{T}=0
$$

and in this case $D_{T *}$ is an orthogonal projection. A direct calculation shows that $\operatorname{det}\left(\lambda I_{n}-D_{T^{*}}^{2}\right)=\lambda(\lambda-1)^{n-1}$ such that the eigenvalue $\lambda=0$ has unit multiplicity and the eigenvalue $\lambda=1$ is degenerated. From the second relation (7) we have

$$
D_{T^{*}} T=D_{T^{*}} b=T D_{T}=b D_{T}=0
$$


i.e. $b$ is the eigenvector of $D_{T^{*}}$ corresponding to $\lambda=0$ eigenvalue.

The orthogonal matrix $\mathcal{O}_{n}$ which brings the operator $D_{T^{*}}$ to a diagonal form

$$
\mathcal{O}_{n}^{t} D_{T^{*}} \mathcal{O}_{n}=\left(\begin{array}{cc}
0 & 0 \\
0 & I_{n-1}
\end{array}\right)
$$

is the orthogonal matrix we are looking for because it is generated by an arbitrary $n$-dimensional real vector of unit norm. The multiplicity of $\lambda=1$ eigenvalue being $n-1$ the form of the matrix $\mathcal{O}_{n}$ is not uniquely defined. Indeed if $v_{1}, \ldots, v_{n}$ are the eigenvectors of $D_{T^{*}}$

$$
D_{T^{*}} v_{1}=D_{T^{*}} b=0, \quad D_{T^{*}} v_{k}=v_{k}, \quad k=2, \ldots, n
$$

orthogonal eigenvectors will be also the vectors

$$
v_{1} \text { and } a_{k}=R v_{k}, \quad k=2,3, \ldots, n
$$

where $R \in O(n)$ is an arbitrary rotation acting only on the last $n-1$ eigenvectors. Thus there is a continuum of solutions for the orthogonal basis that diagonalizes $D_{T^{*}}$. In this situation we have to make a choice between the possible bases. Our criteria was that the resulting orthogonal matrix $\mathcal{O}_{n}$ should have as many as possible vanishing entries. We found such a matrix that have $(n-1)(n-2) / 2$ null entries and the result is expressed by the following lemma:

Lemma 1: The eigenvectors of the eigenvalue problem Eq.(8) which are the columns of the orthogonal matrix $\mathcal{O}_{n} \in S O(n)$ generated by the vector parametrized by Eq.(6) are given by

$$
\begin{gathered}
v_{1}=\left(\begin{array}{l}
\cos \theta_{1} \\
\sin \theta_{1} \cos \theta_{2} \\
\cdot \\
\cdot \\
\cdot \\
\sin \theta_{1} \ldots \sin \theta_{n-1}
\end{array}\right), v_{2}=\left(\begin{array}{l}
-\sin \theta_{1} \\
\cos \theta_{1} \cos \theta_{2} \\
\cos \theta_{1} \sin \theta_{2} \cos \theta_{3} \\
\cdot \\
\cdot \\
\cos \theta_{1} \sin \theta_{2} \ldots \sin \theta_{n-1}
\end{array}\right) \\
v_{k+2}=\left(\begin{array}{l}
0_{k} \\
-\sin \theta_{k+1} \\
\cos \theta_{k+1} \cos \theta_{k+2} \\
\cdot \\
\cdot \\
\cos \theta_{k+1} \sin \theta_{k+2} \ldots \sin \theta_{n-1}
\end{array}\right), \quad k=1, \ldots, n-2
\end{gathered}
$$

where $0_{k}$ means that all the first $k$ entries are zero and $k=1,2, \ldots n-2$. Alternatively

$$
v_{k+1}=\frac{d}{d \theta_{k}} v_{1}\left(\theta_{1}=\cdots=\theta_{k-1}=\pi / 2\right) \quad k=1, \ldots, n-1
$$


The full $O(n)$ group is obtained by multiplying $\mathcal{O}_{n}$ given by Eqs.(9) with the diagonal matrix which has all the entries but the last one 1 and $d_{n, n}=-1$.

Proof: Elementary checking shows that $\left(v_{i}, v_{j}\right)=\delta_{i j}, i, j=1, \ldots, n$, and thus $v_{k}$ are linearly independent. Because the multiplicity of the null eigenvalue is unity it follows that $v_{k}, k=2, \ldots, n$, are orthogonal eigenvectors corresponding to $\lambda=1$ eigenvalue.

Lemma 2 The orthogonal matrices $\mathcal{O}_{n}\left(\mathcal{O}_{n-k}^{k}\right)$ at their turn can be factored into a product of $n-1\left(n\right.$-k-1) matrices of the form $J_{i, i+1}$; e.g. we have

$$
\mathcal{O}_{n}=J_{n-1, n} J_{n-2, n-1} \ldots J_{1,2}
$$

where $J_{i, i+1}$ are $n \times n$ rotations introduced by Eq.(6).

Remark. If the angles that parametrize $\mathcal{O}_{n}$ are $\theta_{1}, \ldots, \theta_{n-1}$, then the angles that parametrize $\mathcal{O}_{n-1}^{1}$ are denoted e.g. by $\theta_{n}, \ldots, \theta_{2 n-3}$, etc. and the last angle entering $\mathcal{O}_{2}^{n-1}$ will be $\theta_{n(n-1) / 2}$.

Putting together all the preceding information one obtains the following result

Theorem: Any element $A_{n} \in U(n)$ can be factored into an ordered product of $2 n-1$ matrices of the following form

$$
A_{n}=d_{n} \mathcal{O}_{n} d_{n-1}^{1} \mathcal{O}_{n-1}^{1} \ldots d_{2}^{n-2} \mathcal{O}_{2}^{n-1} d_{1}^{n-1}
$$

where $d_{n-k}^{k}$ are diagonal matrices and $\mathcal{O}_{n-k}^{k}$ orthogonal matrices whose columns are given by formulae like (9) generated by real $(n-k)$-dimensional unit vectors. Using factorization (10) the above formula can be written as a product of $n$ diagonal matrices and of $n(n-1) / 2$ rotations $J_{k, k+1}$.

The condition $\sum_{i=1}^{n(n+1) / 2} \varphi_{i}=0$, imposed on $\varphi_{i}$ the arbitrary phases entering the parametrization of $A_{n}$, gives the factorization of $S U(n)$ matrices.

$$
\begin{gathered}
\text { If } w_{n}=\mathcal{O}_{n} d_{n-1}^{1} \mathcal{O}_{n-1}^{1} \ldots d_{2}^{n-2} \mathcal{O}_{2}^{n-1} d_{1}^{n-1}=\mathcal{O}_{n} d_{n-1}^{1} w_{n-1} \text { then } \\
W_{n}=w_{n}^{*} d_{n} w_{n}
\end{gathered}
$$

is one (of the many possible) Weyl representation of unitary matrices.

If all the phases entering $A_{n}$ are zero $\varphi_{i}=0, i=1, \ldots, n(n+1) / 2$, one gets the factorization of rotations $R_{n} \in S O(n)$

$$
R_{n}=\mathcal{O}_{n} \mathcal{O}_{n-1}^{1} \ldots \mathcal{O}_{2}^{n-1}
$$

and the full group $O(n)$ is obtained by multiplying (12) with a diagonal matrix $d=(1, \ldots, 1,-1)$ that has one entry equal to -1 .

Remark. The above factorization is not unique and we propose it as the standard (and simplest) representation. Equivalent factorizations (parametrizations) can be obtained by inserting matrices like $P_{i j}$ as factors in the formulae (10)-(12) since the number of parameters remains the same and only the final form of the matrices will be different. As concerns Eq.(11) we made the choice that leads to 
the simplest form for the matrix elements of $W_{n}$ as polynomial functions of sines and cosines which enter the parametrization of orthogonal matrices. For example instead of $w_{n}=\mathcal{O}_{n} d_{n-1}^{1} w_{n-1}$ we could take $w_{n}=\mathcal{O}_{n} W_{n-1}$, where $W_{n-1}$ is at its turn given by a formula like Eq.(11) and so on.

Examples. An element $A_{4} \in U(4)$ factors as

$$
A_{4}=d_{4} \mathcal{O}_{4} d_{3}^{1} \mathcal{O}_{3}^{1} d_{2}^{2} \mathcal{O}_{2}^{2} d_{1}^{3}
$$

where $d_{4}=\left(e^{i \varphi_{1}}, e^{i \varphi_{2}}, e^{i \varphi_{3}}, e^{i \varphi_{4}}\right) d_{3}^{1}=\left(1, e^{i \varphi_{5}}, e^{i \varphi_{6}}, e^{i \varphi_{7}}\right) d_{2}^{2}=\left(1,1, e^{i \varphi_{8}}, e^{i \varphi_{9}}\right), d_{1}^{3}=$ $\left(1,1,1, e^{i \varphi_{10}}\right)$ and $\mathcal{O}_{4}, \mathcal{O}_{3}^{1}$ and $\mathcal{O}_{2}^{2}$ are the following matrices

$$
\begin{gathered}
\mathcal{O}_{4}=\left(\begin{array}{llcc}
\cos \theta_{1} & -\sin \theta_{1} & 0 & 0 \\
\sin \theta_{1} \cos \theta_{2} & \cos \theta_{1} \cos \theta_{2} & -\sin \theta_{2} & 0 \\
\sin \theta_{1} \sin \theta_{2} \cos \theta_{3} & \cos \theta_{1} \sin \theta_{2} \cos \theta_{3} & \cos \theta_{2} \cos \theta_{3} & -\sin \theta_{3} \\
\sin \theta_{1} \sin \theta_{2} \sin \theta_{3} & \cos \theta_{1} \sin \theta_{2} \sin \theta_{3} & \cos \theta_{2} \sin \theta_{3} & \cos \theta_{3}
\end{array}\right) \\
\mathcal{O}_{3}^{1}=\left(\begin{array}{cccc}
1 & 0 & 0 & 0 \\
0 & \cos \theta_{4} & -\sin \theta_{4} & 0 \\
0 & \sin \theta_{4} \cos \theta_{5} & \cos \theta_{4} \cos \theta_{5} & -\sin \theta_{5} \\
0 & \sin \theta_{4} \sin \theta_{5} & \cos \theta_{4} \sin \theta_{5} & \cos \theta_{5}
\end{array}\right) \\
\mathcal{O}_{2}^{2}=\left(\begin{array}{cccc}
1 & 0 & 0 & 0 \\
0 & 1 & 0 & 0 \\
0 & 0 & \cos \theta_{6} & -\sin \theta_{6} \\
0 & 0 & \sin \theta_{6} & \cos \theta_{6}
\end{array}\right)
\end{gathered}
$$

The formula (10) for $\mathcal{O}_{4}$ is

$$
\begin{gathered}
\mathcal{O}_{4}=J_{3,4} \cdot J_{2,3} \cdot J_{1,2}= \\
\left(\begin{array}{cccc}
1 & 0 & 0 & 0 \\
0 & 1 & 0 & 0 \\
0 & 0 & \cos \theta_{3} & -\sin \theta_{3} \\
0 & 0 & \sin \theta_{3} & \cos \theta_{3}
\end{array}\right)\left(\begin{array}{cccc}
1 & 0 & 0 & 0 \\
0 & \cos \theta_{2} & -\sin \theta_{2} & 0 \\
0 & \sin \theta_{2} & \cos \theta_{2} & 0 \\
0 & 0 & 0 & 1
\end{array}\right)\left(\begin{array}{cccc}
\cos \theta_{1} & -\sin \theta_{1} & 0 & 0 \\
\sin \theta_{1} & \cos \theta_{1} & 0 & 0 \\
0 & 0 & 1 & 0 \\
0 & 0 & 0 & 1
\end{array}\right)
\end{gathered}
$$

The Weyl form of a $2 \times 2$ unitary matrix is

$$
\begin{gathered}
W_{2}=w_{2}^{*} d_{2} w_{2}=d_{1}^{1^{*}} \mathcal{O}_{2}^{t} d_{2} \mathcal{O}_{2} d_{1}^{1}= \\
\left(\begin{array}{cc}
e^{i \varphi_{1}} \cos ^{2} \theta+e^{i \varphi_{2}} \sin ^{2} \theta & \cos \theta \sin \theta e^{i \varphi_{3}}\left(e^{i \varphi_{1}}-e^{i \varphi_{2}}\right) \\
\cos \theta \sin \theta e^{-i \varphi_{3}}\left(e^{i \varphi_{1}}-e^{i \varphi_{2}}\right) & e^{i \varphi_{2}} \cos ^{2} \theta+e^{i \varphi_{1}} \sin ^{2} \theta
\end{array}\right)
\end{gathered}
$$

where $d_{2}=\left(e^{i \varphi_{1}}, e^{i \varphi_{2}}\right), d_{1}^{1}=\left(1, e^{i \varphi_{3}}\right)$ and $\mathcal{O}_{2}=U$, and $U$ is the matrix (1). 


\section{Concluding remarks}

In this paper we proposed a new factorization of unitary matrices which can be useful in many domains of mathematical and theoretical physics. For example the construction of coherent states for $S U(n)$ can be developed similarly to that for the $S U(3)$ group given in [0]. The $S U(n)$ matrices are obtained from the

above parametrization by imposing the condition $\sum_{i=1}^{i=n(n-1)} \varphi_{i}=0$ upon the phases and let us denote by the same letter $A_{n}$ an arbitrary element of $S U(n)$. We consider the first $n-1$ columns of $A_{n}$ as vectors $v_{k}$ whose entries are given by

$$
\left(v_{k}\right)_{i}=A_{i, k}, \quad i=1, \ldots, n, k=1, \ldots, n-1
$$

and consider a set of $n(n-1)$ annihilation operators that we view as the components of $(n-1)$ annihilation vector operators

$$
\left(a_{k}\right)_{i}=a_{i k}, \quad i=1, \ldots, n, k=1, \ldots, n-1
$$

with the usual commutation relations

$$
\left[a_{i k}, a_{j l}\right]=0, \quad\left[a_{i k}, a_{j l}^{\dagger}\right]=\delta_{i j} \delta_{k l}
$$

The key element in defining coherent states is the generating function

$$
\left|v_{1}, \ldots, v_{n-1}>_{n_{1}, \ldots, n_{n-1}}=\sqrt{n_{1} ! \ldots n_{n-1} !} \exp \sum_{1}^{n-1}\left(a_{i}^{\dagger}, v_{i}\right)\right| 0>
$$

where $n_{1}, \ldots, n_{n-1}$ are the integers that index the representation, $\mid 0>$ is the vacuum vector and we used the standard notation $\uparrow$ for the adjoint of the annihilation operator.

Another problem could be the finding of Laplace-Beltrami operators on unitary groups that is an old problem [14]. The Laplace-Beltrami operator on $S_{2 n-1}$ can be written easily as

$$
\begin{gathered}
\Delta=\sum_{k=1}^{n-1} \frac{1}{\sin ^{2} \theta_{1} \ldots \sin ^{2} \theta_{k-1} \cos \theta_{k} \sin ^{2(n-k)-1} \theta_{k}} \frac{\partial}{\partial \theta_{k}}\left(\cos \theta_{k} \sin ^{2(n-k)-1} \theta_{k} \frac{\partial}{\partial \theta_{k}}\right)+ \\
\sum_{k=1}^{n-1} \frac{1}{\sin ^{2} \theta_{1} \ldots \sin ^{2} \theta_{k-1} \cos ^{2} \theta_{k}} \frac{\partial^{2}}{\partial \varphi_{k}^{2}}+\frac{1}{\sin ^{2} \theta_{1} \ldots \sin ^{2} \theta_{n-1}} \frac{\partial^{2}}{\partial \varphi_{n}^{2}}
\end{gathered}
$$

where we used the parametrisation $v_{n}=\left(e^{\varphi_{1}} \cos \theta_{1}, \ldots, e^{\varphi_{n}} \sin \theta_{1} \ldots \sin \theta_{n-1}\right) \in$ $S_{2 n-1}$ With factorization (10) the mathematical tractability problem for $S U(3)$ [14] and other unitary groups can be resolved. A complete treatment of such problems will be given elsewhere. 
Our proposal is largely based on several simple ideas suggested earlier by many people and we have written the factorization in the simplest possible way. We suggest it to become the standard one since any other existing parametrization can be brought to this form by multiplying with permutation matrices like $P_{i j}$ and/or diagonal matrices with entries containing phases.

\section{Acknowledgments}

The author acknowledges a partial financial support of the Rumanian Academy through the grant No.49/2000.

\section{References}

[1] F.D. Murnagham, The Unitary and Rotation Groups, (1962), Spartan Books, Washington, D.C.

[2] H. Harari and M. Leurer, Recommending s Standard Choice of Cabbibo Angles and KM Phases for Any Number of Generators, Phys. Lett. B181 (1986) $123-128$

[3] M. Reck, A. Zeilinger, H.J. Bernstein and P. Bertani, Experimental Realization of Any Discrete Unitary Operator, Phys.Rev.Lett. 73 (1994) 58-61

[4] D.J. Rowe, B.C. Sanders and H. de Guise, Representations of the Weyl Group and Wigner Functions for SU(3), J.Math.Phys. 40 (1999) 3604-3615

[5] Kae Nemoto, Generalised Coherent States for $S U(n)$ Systems, qunt$\mathrm{ph} / 0004087$

[6] S. Chaturvedi and N. Mukunda, Parametrizing the Mixing Matrix: a Unified Approach, hep-ph/0004219

[7] M Mathur and D Sen, Coherent States For SU(3), quant-ph/0012099

[8] H. Weyl, The Classical Groups, (1946), Princeton University Press, New Jersey

[9] S.R. Wadia, $N=\infty$ Phase Transition in a Class of Exactly Soluble Model Lattice Gauge Theories, Phys.Lett. 93B (1980) 403-410

[10] P. Menotti and E. Onofri, The Action of $S U(N)$ Lattice Gauge Theory in terms of the Heat Kernel on the Group Manifold, Nucl.Phys. B190[FS3] (1981) 288-300

[11] P.Diţă, On the Parametrisation of Unitary Matrices by the Moduli of their Elements, Commun.Math.Phys. 159 (1994) 581 
[12] B. Sz-Nagy and C. Foias, Analyse Harmonique des Opérateurs de l'Espace de Hilbert, Masson, Paris, 1967

[13] K. Nemoto, Generalised Coherent States for SU(n) Systems, quant$\mathrm{ph} / 0004087$

[14] M.A.B. Bég and H.Ruegg, A set of Harmonic Functions for the Group SU(3), J.Math.Phys. 6 (1965) 677-682 